\title{
Could a Step in the Isolation of Mycobacteria from Sputum Samples be Eliminated by New Decontamination Kits?
}

\author{
ENGIN KARAKEÇE*, HÜSEYIN AGAH TERZI and İHSAN HAKKI ÇIFTCI
}

Department of Medical Microbiology, Sakarya University, School of Medicine, Sakarya, Turkey

Submitted 17 March 2014, revised 28 June 2014, accepted 15 July 2014

\begin{abstract}
The efficacy of two mycobacterial homogenization, decontamination and concentration kits, Mycoprosafe and Decomics, were compared. A total of 146 sputum samples were examined in this study; 46 (31.5\%) mycobacterial isolates were recovered with Mycoprosafe, while 39 (26.7\%) mycobacterial isolates were recovered with Decomics. These results, although very preliminary, indicate that Mycoprosafe and Decomics are similar in terms of ease of use and price. However, Decomics offers several advantages, including the need for less equipment, a more rapid detection time, and an easy shipping process; thus, pre-processing can be done anywhere.
\end{abstract}

Key words: mycobacteria, sputum samples, decontamination

The administration of appropriate antibiotic therapy for tuberculosis requires that a microbiological diagnosis, consisting of identification of the causative agent and its resistance profile, is obtained. The gold standard for tuberculosis diagnoses remains culture (Kurtoğlu et al., 2011). However, sputum must be pretreated for culture in all media, as compared to other clinical samples. Because sputum samples pass through the oropharynx during collection, culture contamination limits the diagnostic yield. Therefore, many techniques have been developed to improve sputum culture for the diagnosis of tuberculosis. Among these procedures, NALC-NaOH is the standard technique for homogenisation-decontamination-concentration (HDC) (Peres et al., 2009).

Different methods for HDC are used worldwide, some of which were specifically developed with the aim of creating a simple, effective, and low-cost technique. However, in routine clinical practice, HDC methods have certain disadvantages: they kill many mycobacteria, the procedures are tedious and time-consuming, and there is an increased risk of specimen contamination leading to culture contamination rates of 5-9\%, which decreases the sensitivity (Maciel et al., 2009; Rivas et al., 2010). Few published studies have addressed the best and fastest method for sputum decontamination in the diagnosis of tuberculosis (Peres et al., 2009; Ganoza et al., 2008; Martin et al., 1968).
Mycoprosafe (Salubris Inc., Istanbul, Turkey) contains all of the materials needed for application of the classical NALC-NaOH HDC method. Decomics (Salubris Inc.Istanbul, Turkey) is a newly released kit that removes decontamination and neutralisation fluids using absorbent beads and thus eliminates the need for centrifugation. Both Mycoprosafe and Decomics can be kept at room temperature for several days without alteration. The final pellet obtained from both methods can also be stored for later use. However, at first glance Decomics appears to be simpler to use than Mycoprosafe; it saves time, offers reliable shipping, and requires the fewest pieces of laboratory equipment.

The efficacy of two mycobacterial homogenization, decontamination, and concentration kits, Mycoprosafe and Decomics, were compared. Efficacy was determined based upon the rate of successful mycobacterial isolation, the degree of residual contamination, and the time to detection of mycobacteria in liquid culture. Samples were obtained between February and May of 2013 at the Education and ResearchHospital (Sakarya, Turkey) from patients with suspected pulmonary tuberculosis, with or without a previous diagnosis of pulmonary tuberculosis, and before starting appropriate anti-tuberculosis treatment. The procedures were performed at a Biological Safety Level 3 facility in the hospital's Mycobacteriology Laboratory. All samples were stored at $4^{\circ} \mathrm{C}$, and processed within $24 \mathrm{~h}$ of their

\footnotetext{
* Corresponding author: E. Karakeçe, Department of Medical Microbiology, Sakarya University, School of Medicine, Sakarya, Turkey; phone: +90264 25521 10; fax: +90264 255 202; e-mail: enginkarakece@gmail.com
} 
arrival at the laboratory. Two $1 \mathrm{~mL}$ aliquots were taken from each homogenized sample, and processed using either the Mycoprosafe or Decomics kits, according to the manufacturer's instructions. Samples were then inoculated into an appropriate liquid media for detection of mycobacteria. Samples with a volume $<2 \mathrm{~mL}$ were excluded from this analysis

The samples were processed with Mycoprosafe and Decomics according to the manufacturer's recommendations (Kubica et al., 1963). Each processed sample was examined by acid-fast bacilli (AFB) staining with Ziehl-Neelsen stain. The AFB staining results were reported as follows: $<1+, 1-9$ AFB in 100 microscopic fields (few bacilli); 1+, 10-99 AFB in 100 fields; 2+, 1-10 AFB per field in at least 50 fields; $3+,>10$ AFB per field in at least 20 fields.

After the HDC process, the resuspended pellets were inoculated according to the manufacturer's recommendations for TK-SLC-L (Salubris Inc.) and MGIT (Becton Dickinson) culture tubes and incubated in the Mycolor TK (Salubris Inc.) and BACTEC MGIT 960 (Becton Dickinson) instruments, respectively. Growth signals were confirmed by AFB staining and a TBc ID (Becton Dickinson) identification kit, which was used according to the manufacturer's recommendations. Contamination control for the MGIT system was done by subculturing on sheep blood agar; contamination was followed using the Mycolor TK system, which can predict contamination as well as growth. All data were analysed using SPSS (version 17.0); frequency, cross-tab, and McNemar tests were used. A total of 146 sputum samples were examined in this study. Of these, $11(7.5 \%)$ specimens with very low acid-fast bacilli (AFB) counts $(<1+)$ were misdiagnosed as positive by AFB staining using both the Mycoprosafe and Decomics kits. Mycobacterial isolation rate were fond 46 (31.5\%) with Mycoprosafe while were found 39 (26.7\%) with Decomics. All positive cultures were identified as Mycobacterium tuberculosis. The cultures were less frequently contaminated in the MGIT system after processing with Decomics, as compared to Myco- prosafe; however, the difference was not significant. In contrast, the cultures were contaminated less frequently after processing with Mycoprosafe, as compared to Decomics in the TK-SLC-L liquid culture system, and the difference was significant $(\mathrm{p}=0.041)$.

Additionally, there was a difference between the HDC kits in terms of the average time to detection of growth for both automated culture systems. The time to detection of growth varied between 5.2 and 34.8 days with the TK-SLC-L system and between 2.0 and 34.1 days with the MGIT system for specimens that were processed with Mycoprosafe. The time to detection of growth varied between 4.1 and 31.1 days with the TK-SLC-L system and between 2.0 and 30.1 days with the MGIT system for specimens that were processed with Decomics. The microscopy, culture, and time to detection of growth results obtained for samples processed using Mycoprosafe and Decomics are summarised in Table I.

Accurate case detection is essential for the control of short-course tuberculosis. Many techniques have been developed to improve the sensitivity and timely detection of AFB in sputum. However, not much progress has been made in terms of sample preparation protocols. Additionally, the processes used currently for decontamination and homogenisation to facilitate the isolation of mycobacteria may kill mycobacterial cells. Even with a relatively mild method, the loss of mycobacteria could be as high as $10^{4}$ depending on the species present (Ratnam et al., 1987). Therefore, care should be taken in selecting an HDC technique.

Previous work examining serial smears from 15,821 suspected tuberculosis (TB) patients found that the majority of cases (91.6\%) were detected in the first smear. A second smear identified an additional 303 cases $(7.4 \%)$, with a third smear detecting 42 (1\%) cases (Yassin and Cuevas, 2003). These results suggest that the vast majority of infections (99\%) can be detected with only two serial smears. In this study, microscopic examinations identified far fewer cases in suspected TB patients than previously reported, which

Table I

Results from samples processed using two different HDC methods

\begin{tabular}{|l|c|c|c|c|c|c|}
\hline \multirow{2}{*}{} & \multicolumn{2}{|c|}{ Mycoprosafe } & \multicolumn{3}{c|}{ Decomics } \\
\cline { 2 - 7 } & $\begin{array}{c}\text { MGIT } \\
\mathrm{n} / \mathrm{N}(\%)\end{array}$ & $\begin{array}{c}\text { TK-SLC-L } \\
\mathrm{n} / \mathrm{N}(\%)\end{array}$ & $\mathrm{p}$ & $\begin{array}{c}\text { MGIT } \\
\mathrm{n} / \mathrm{N}(\%)\end{array}$ & $\begin{array}{c}\text { TK-SLC-L } \\
\mathrm{n} / \mathrm{N}(\%)\end{array}$ & $\mathrm{p}$ \\
\hline Isolation & $34 / 146(23.3)$ & $35 / 146(24.0)$ & $>0.05$ & $31 / 146(21.2)$ & $33 / 146(22.6)$ & $>0.05$ \\
\hline Among total isolation & $34 / 46(73.9)$ & $35 / 46(76.1)$ & $>0.05$ & $31 / 39(79.5)$ & $33 / 39(84.6)$ & $>0.05$ \\
\hline Contamination & $20 / 54(37.0)$ & $2 / 37(5.4)$ & 0.041 & $14 / 45(31.1)$ & $9 / 42(21.4)$ & $>0.05$ \\
\hline Mean growth time (days) & 13.1 & 18.3 & $>0.05$ & 10.5 & 13.9 & $>0.05$ \\
\hline Median growth time (days) & 7.9 & 15.1 & $>0.05$ & 7.7 & \multicolumn{3}{|c|}{$39 / 146(26.7)$} & $>0.05$ \\
\hline Total culture positivity [n/N (\%)] & \multicolumn{3}{|c|}{$46 / 146(31.5)$} & & \multicolumn{4}{c}{} \\
\hline
\end{tabular}


could potentially lead to delayed diagnosis and therapy. This discrepancy is likely to be the result of differences in sample collection practices, and the low bacillary count $(<1+)$ in many of the samples examined (Yassin and Cuevas, 2003).

Decontamination failures are often associated with user error; however the simplicity of the collection kits should be sufficient to eliminate most handling errors. Previous studies have reported contamination rates for liquid culture systems between 10.0-20.1\%, similar to the results presented here. While decontamination and concentration of the samples was performed by the same technicians, contamination was significantly less frequent when using the Mycoprosafe kit, as compared to the Decomics kit $(\mathrm{p}<0.041)$. This difference may have arisen as a result of the extra manipulation steps associated with the MGIT system, or as a result of suboptimal bacterial concentration in the starting material. This discordance in contamination rates may be solved by adjusting the concentration of $\mathrm{NaOH}$ used.

Total growth in a liquid culture system was found in 46 (31.6\%) and 39 (26.7\%) samples with the Mycoprosafe and Decomics HDC kits, respectively. Slightly lower isolation rates were obtained in samples processed using the Decomics kit. However, it is unclear at which step the preliminary preparation protocol failed or which samples were most likely to be affected. This situation may be due to the higher contamination rates associated with the TK-SLC-L system. Other possible reasons are toxicity, a loss of bacilli from the absorbent beads, and the presence of damaged bacilli.

The contamination count was lower for the MGIT system than for the TK-SLC-L system with samples prepared using Decomics, as compared to those prepared using Mycoprosafe. This may have been due to processing errors. The contamination rate may be lowered by changing the decontamination time from 10 to $15 \mathrm{~min}$, as recommended in the Decomics manual.

Decomics speeds up the mycobacterial detection time by 3 to 5 days depending on the liquid culture method used. Accurate case detection is essential for the treatment of short-course tuberculosis. All tuberculosis control strategies focus on early detection to decrease transmission. Furthermore, Decomics, which lowers the sample processing time from 45 to $25 \mathrm{~min}$ and eliminates the need for centrifugation, may be a good alternative to the classical Kubica method in laboratories with elaborate capabilities. It may also enable laboratories where equipment and methods such as centrifugation are limited to do HDC and, thus, mycobacterial culture.
According to our results, which are very preliminary, Mycoprosafe and Decomics were similar in terms of their ease of use and price. Decomics appears to have an advantage because it requires less equipment and offers both a faster detection time and easier shipping, which means that it can be easier and practical to apply in routine use. However, the isolation rate using Decomics was slightly lower. If this problem is resolved, Decomics could be of great help in increasing the diagnosis of tuberculosis in developing country settings.

\section{Acknowledgments}

This is an experimental study. In this study, in vitro samples were investigated prospectively. Extra samples were not taken from any patient.

\section{Literature}

Ganoza C.A., J.N. Ricaldi, J. Chauca, G. Rojas, C. Munayco, J. Agapito, J.C. Palomino and H. Guerra. 2008. Novel hypertonic saline-sodium hydroxide (HS-SH) method for decontamination and concentration of sputum samples for Mycobacterium tuberculosis microscopy and culture. J. Med. Microbiol. 57: 1094-1098.

Kubica G.P., W.E. Dye, M.L. Cohn and G. Middlebrook. 1963. Sputum digestion and decontamination with $\mathrm{N}$-acetyl-L-cysteinesodium hydroxide for culture of mycobacteria. Am. Rev. Respir. Dis. 89: 284-286.

Kurtoğlu M.G., M. Ozdemir, R. Keşli, B. Ozkalp and B. Baysal. 2011. Tüberküloz Şüpheli Hastalardan Mycobacterium tuberculosis Kompleks İzolasyon Oranı ve Suşların BACTEC ${ }^{\mathrm{TM}}$ NAP ve İmmünokromatografik TB Ag MPT64 Rapid ${ }^{\mathrm{TM} T e s t l e r i}$ ile Tanımlanması. Mikrobiyol. Bul. 45(2): 266-273.

Maciel E.L., T.N. Prado, R.L. Peres, M. Palaci, J.L. Johnson and R. Dietze. 2009. Guided sputum sample collection and culture contamination rates in the diagnosis of pulmonary. TB. J. Bras. Pneumol. 35(5): 460-463.

Martin M.E., C.L. Horton, A.L. Sheffner and J.D. Solonon. 1968. Direct comparison of the $\mathrm{N}$-acetyl-L-cysteine-sodium hydroxide and the trisodium phosphate digestion methods for the culture of mycobacteria. Appl. Microbiol. 16: 506-508.

Peres R.L., E.L. Maciel, C.G. Morais, F.C.K. Ribeiro, S.A. Vinhas, C. Pinheiro, R. Dietze, J.L. Johnson, K. Eisenach and M. Palaci. 2009. Comparison of two concentrations of NALC-NaOH for decontamination of sputum for mycobacterial culture. Int. J. Tuberc. Lung. Dis. 13(12): 1572-1575.

Ratnam S., F.A. Stead and M. Howes. 1987. Simplified acetylcysteine-alkali digestion-decontamination procedure for isolation of mycobacteria from clinical specimens. Clin. Microbiol. 25(8): $1428-1432$.

Rivas C., C. Coitinho, V. Dafond, M. Corbo and M. Baldjian. 2010. Performance of the Ogawa-Kudoh method for isolation of mycobacteria in a laboratory with large-scale workload. Rev. Argent. Microbiol. 42(2): 87-90.

Yassin M.A. and L.E. Cuevas. 2003. How many sputum smears are necessary for case finding in pulmonary tuberculosis? Tropical Medicine and International Health 8 (10): 927-932. 
